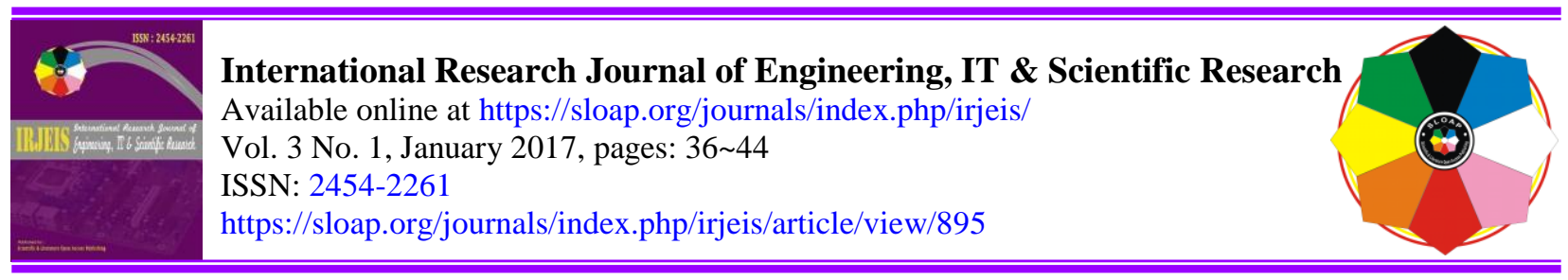

\title{
Automatic Classification of MR Brain Tumor Images using KNN, ANN, SVM and CNN
}

Hema Rajini $\mathbf{N}^{\text {a }}$

Article history:

Received: 18 August 2016

Accepted: 27 November 2016

Published: 31 January 2017

\section{Keywords:}

artificial neural network; convolutional neural networks;

k-nearest neighbor;

support vector machine;

\begin{abstract}
A brain tumor classification system has been designed and developed. This work presents a new approach to the automated classification of astrocytoma, medulloblastoma, glioma, glioblastoma multiforme and craniopharyngioma type of brain tumors based on first order statistics and gray level cooccurrence matrix, in magnetic resonance images. The magnetic resonance feature image used for the tumor detection consists of T2-weighted magnetic resonance images for each axial slice through the head. To remove the unwanted noises in the magnetic resonance image, median filtering is used. First order statistics and gray level co-occurrence matrix-based features are extracted. Finally, k-nearest neighbor, artificial neural network, support vector machine and convolutional neural networks are used to classify the brain tumor images. The application of the proposed method for tracking tumor is demonstrated to help pathologists distinguish its type of tumor. A classification with an accuracy of $89 \%, 90 \%, 91 \%$ and $95 \%$ has been obtained by, k-nearest neighbor, artificial neural network, support vector machine and convolutional neural networks.
\end{abstract}

2454-2261 ${ }^{\odot}$ Copyright 2017. The Author. This is an open-access article under the CC BY-SA license (https://creativecommons.org/licenses/by-sa/4.0/) All rights reserved.

\footnotetext{
Author correspondence:

Hema Rajini N,

Department of Computer Science and Engineering, Annamalai University,

Annamalai Nagar - 608002, Tamilnadu, India

Email address : auhemasmith@yahoo.co.in
}

\section{Introduction}

Brain tumor is often a group of abnormal cells due to loss of normal aging and cell death. It may occur in any individual at almost any age. It may also differ from one treatment session to another; however, its effects will not be the same for each person. Brain tumors appear at any place, in different image intensities, can have a number of sizes and shapes. Brain tumors can be benign or malignant; it is categorized according to World Health Organization (WHO) as neuroepithelial tumors, non-neuroepithelial tumors, mutations leading to astrocytic tumors and astrocytic

a Annamalai University, Annamalai Nagar, Tamilnadu, India 
lineage tumor grading systems. In this particular research work astrocytoma, medulloblastoma, glioma, glioblastoma multiforme and craniopharyngioma type of brain tumors are utilized.

There are many computer-aided classification systems for Magnetic Resonance (MR) and Computed Tomography (CT) brain images in the literature, most of them are used to detect and classify abnormalities. Dipali $e t$ al. (2010) proposed a method to detect and classify astrocytoma tumor in MR brain images using thresholding and ANN classifier. Ahmed et al. (2010), proposed a method using wavelet-based texture features. The optimum features are selected using genetic algorithm and given to support vector machine for efficient classification of brain MR images with high sensitivity 94\%, specificity 100\% and accuracy 96\%. Fazel Zarandi et al. (2011), proposed a method for classification of different grades in astrocytoma tumor. Tumor region is segmented using possibilistic cmeans method and feature extraction is done by thresholding method. Finally, approximate reasoning is used to classify the tumor grade in brain MRI. The results showed that the proposed system is superior in classifying the brain tumor and its grade than type I fuzzy expert system.

Chawla et al. (2009), proposed a method to detect and classify the types of stroke in brain CT images. Doi (2005), gave a survey of current status and future potential of computer-aided diagnosis in medical imaging. Gong et al., (2011), proposed a method to classify traumatic brain images using ROI labelling and atlas-based classification. Padma \& Sukanesh (2011), proposed a method to classify and segment CT brain tumour images by using optimal dominant gray level run length texture features. It was compared with wavelet-based texture feature extraction method and spatial gray level dependence matrix method. The optimal features were selected using genetic algorithm. The selected features were fed to the support vector machine (SVM) classifier to classify and segment the tumour brain CT images. The classification accuracy was $98 \%$. Zhang et al. introduced a method based on the features of gray scale, shape and texture, and the symmetric feature human brain CT image is classified (Zhang \& Wang, 2007). Inductive learning techniques, see 5 and radial basis function of nerve network, were used to build classifiers.

Hema Rajini \& Bhavani (2014), proposed a new approach to classify the normal and abnormal images of CT. The proposed method consists of four stages namely pre-processing, feature extraction, feature reduction and classification. The discrete wavelet transform coefficients are the features extracted in this method. The essential coefficients are selected by the principal component analysis. A classification with a success of $92 \%, 97 \%$ and $98 \%$ has been obtained by artificial neural network, k-nearest neighbour and support vector machine, respectively.

The rest of this paper is organized as follows. Section 1 presents the introduction as well as the studies of several research papers are portrayed. Section 2 presents the proposed technique, utilized in this work for classification of astrocytoma, medulloblastoma, glioma, glioblastoma multiforme and craniopharyngioma type MR brain images. In this section, pre-processing, feature extraction and classification are presented. Section 3 experimentally demonstrates the performance of the proposed method. Finally, Section 4 describes the conclusion of this paper.

\section{Materials and Method}

\section{Materials}

The data set of brain MR image was collected from Rajah Muthaiah Medical College Hospital (RMMCH) in Chidambaram to develop our algorithm. The field of view (FOV) is $240 \mathrm{~mm}$. Each image is $512 \times 512$ pixels in size. The proposed system is experimented and tested using 26 positive cases. The thickness of brain MR image is $5 \mathrm{~mm}$. All are performed on a 1.5 T MR scanner (Intera Release 11, Philips medical systems, Nederland B.V). Consecutive Digital Imaging and Communications in Medicine (DICOM) MR slices are imported into the Mat lab workspace automatically.

\section{Proposed Method}

This proposed research work is used to improve brain tumor classification in brain MR images. The proposed method has three stages, namely pre-processing, feature extraction and classification. The proposed technique for automatic MR brain tumor image classification is illustrated in Figure 1. The proposed system is developed using Matlab (The Math Works, Inc., Natick, MA, USA). In the first stage, noise is suppressed using an image filtering. In the second stage first order statistics and gray level co-occurrence matrix-based features are extracted. Finally, KNN, ANN SVM and CNN classifiers are used to classify the type of tumor images.

Rajini N, H. (2017). Automatic classification of MR brain tumor images using KNN, ANN, SVM and CNN. International Research Journal of Engineering, IT \& Scientific Research, 3(1), 36-44. Retrieved from https://sloap.org/journals/index.php/irjeis/article/view/895 
a) Pre-processing

To reduce noise, median filtering using a 3-by-3 square kernel is applied (Fazel Zarandi et al., 2011). Median filter is chosen because it is less sensitive to extreme values and able to remove outliers without reducing sharpness of the image. This produces a more homogeneous background in which abnormalities become more conspicuous.

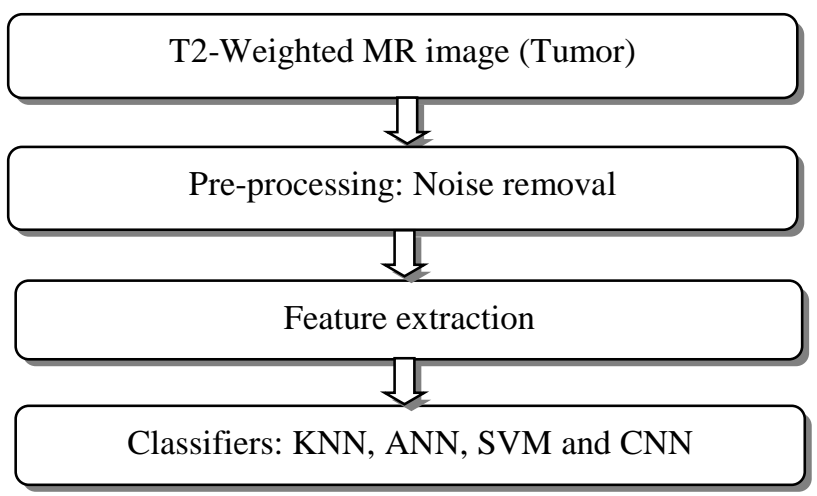

Figure 1. Methodology of the proposed technique

b) Texture Features from Gray Level Co-occurrence Matrix

Texture is a repeating pattern of local variations in image intensity. The co-occurrence matrix is a statistical method used for texture analysis. As the name suggests, the co-occurrence matrix is constructed from the image by estimating the pair wise statistics of pixel intensity. The use of the co-occurrence matrix is based on the hypotheses that the same gray-level configuration is repeated in a texture. This pattern will vary more by fine textures than by coarse textures. The co-occurrence matrix $P(i, j \mid d, \theta)$ counts the co-occurrence of pixels with gray values $i$ and $j$ at a given distance $d$ and in a given direction $\theta$. According to the number of intensity points (pixels) in each combination, statistics are classified into first-order, second-order and higher-order statistics. In the first order, texture measures are statistics calculated from an individual pixel and do not consider pixel neighbor relationships. The gray level co-occurrence matrix (GLCM) method is a way of extracting second order statistical texture features (Haralick et al., 1973). However, the performance of a given GLCM based feature, as well as the ranking of the texture features; depend on the number of gray levels used. We use the following notation: $\mu$ is the mean value of $P . \mu_{x}, \mu_{y}, \sigma_{x}$ and $\sigma_{y}$ are the means and standard deviations of $P_{x}$ and $P_{y} . G$ is the size of the co-occurrence matrix. Here the number of rows and columns of the co-occurrence matrix is equal. In this work mean, standard deviation, skewness and kurtosis are the first order statistics features. The following GLCM based texture features are extracted in this research work: contrast, correlation, energy, homogeneity and entropy. They are defined in eqs. (1)-(5).

Contrast

Contrast $=\sum_{n=0}^{G-1} n^{2}\left\{\sum_{i=1}^{G} \sum_{j=1}^{G} P(i, j)\right\},|i-j|=n$

Contrast is a measure of the local variations present in an image. This measure of contrast favors contributions from $P(i, j)$ away from the diagonal, i.e. $i=j$. If there is a large amount of variations in an image, the $P[i, j]$ 's will be concentrated away from the main diagonal and the contrast will have a high value.

Correlation

Correlation $=\sum_{i=0}^{G-1} \sum_{j=0}^{G-1} \frac{\{i \times j\} \times P(i, j)-\left\{\mu_{x} \times \mu_{y}\right\}}{\sigma_{x} \times \sigma_{y}}$

Correlation is a measure of gray level linear dependence between the pixels at the specified positions relative to 
each other. The correlation will be higher if an image contains a considerable amount of linear structure.

$$
\begin{aligned}
& \text { Energy } \\
& \text { Energy }=\sum_{i=0}^{G-1} \sum_{j=0}^{G-1} p(i, j)^{2}
\end{aligned}
$$

The energy of a texture describes the uniformity of the texture. Energy is 1 for a constant image.

Homogeneity

$$
\text { Homogeneity }=\sum_{i=0}^{G-1} \sum_{j=0}^{G-1} \frac{P(i, j)}{1+|i-j|}
$$

Homogeneity returns a value that measures the closeness of the distribution of elements in the GLCM to the GLCM diagonal. Homogeneity is 1 for a diagonal GLCM. A homogeneous image will result in a co-occurrence matrix with a combination of high and low $P[i, j]$ 's. A heterogeneous image will result in an even spread of $P$ $[i, j]$ 's.

Entropy
Entropy $=-\sum_{i=0}^{G-1} \sum_{j=0}^{G-1} P(i, j) \times \log (P(i, j))$

Entropy statistic measures the disorder or complexity of an image. Complex textures tend to have high entropy. Entropy is strongly, but inversely correlated to energy.

c) Classifiers: KNN, ANN, SVM and CNN

Classification is the process of classifying the given input by training with a suitable classifier. Deep learning and Support Vector Machine (SVM) classifiers are the best classifiers suggested by many researchers which can be opted for the brain tumor classification of MR images. It is independent of dimensionality and feature space. Convolutional Neural Networks (CNN) is one of the most remarkable approaches of deep learning, in which multiple layers of neurons are formed in a robust manner. In this work simple classifiers like KNN and ANN are also used.

k-Nearest neighbor classifier: One of the simplest classification techniques is the k-nearest neighbor (k-NN) classifier. Classification of the input feature vector $\mathrm{X}$ is done by determining the $\mathrm{k}$ closest training vectors according to a suitable distance metric. Vector $\mathrm{X}$ is then assigned to that class to which the majority of that $\mathrm{k}-$ nearest neighbors belong. The k-NN algorithm is based on a distance function and a voting function in k-nearest neighbors; the metric employed is the Euclidean distance measure (Cover \& Hart, 1967). The k-NN classifier is a conventional nonparametric supervised classifier that is said to yield good performance for optimal values of $\mathrm{k}$. Like most learning algorithms, k-NN algorithm consists of a training phase and a testing phase. Data points are given in an n-dimensional space in the training phase. The labels associated with the data points designate their class in the training phase. In the testing phase, unlabeled data are given and the algorithm generates the list of the k-nearest (already classified) data points to the unlabeled point. This classifier returns the class of the majority of that list.

Artificial Neural Network Classifier: An ANN is a mathematical model consisting of a number of highly interconnected processing elements organized into layers, geometry and functionality of which have been resembled to that of the human brain. The ANN may be regarded as a massively parallel distributed processor that has a natural propensity for storing experiential knowledge and making it available for use (Baxt, 1995). The cascade-forward back propagation network which was employed as the classifier required in this study had two layers (after several trails for different hidden layers with a different number of neurons). The first layer consists of 14 input elements in accordance with the 9 feature vectors selected from the texture features by first order statistics and GLCM. The number of neurons in the hidden layer is ten and thirty. The single neuron in the output layer is used to represent the type of brain tumor. The neural network has been trained to adjust the connection

Rajini N, H. (2017). Automatic classification of MR brain tumor images using KNN, ANN, SVM and CNN. International Research Journal of Engineering, IT \& Scientific Research, 3(1), 36-44. Retrieved from https://sloap.org/journals/index.php/irjeis/article/view/895 
weights and biases in order to produce the desired mapping. In this stage, the feature vectors are applied as an input to the network and the network adjusts its variable parameters, the weights and biases, to capture the relationship between the input patterns and outputs.

Support Vector Machine Classifier: Support vector machine (SVM) is a powerful supervised classifier and accurate learning technique. From the statistical theory it was derived and developed by Vapnick in 1982. It yields successful classification results in various application domains, e.g. medical diagnosis. SVM is based on the structural risk minimization principle from the statistical learning theory (Cristianini \& Shawe Taylor, 2000). The kernel controls the empirical risk and classification capacity in order to maximize the margin between the classes and minimize the true costs. SVM searches an optimal separating hyper-plane between members and nonmembers of a given class in a higher dimensional feature space. The inputs to the SVM algorithm are the features extracted from first order statistics and GLCM. In our method, multi classes are astrocytoma, medulloblastoma, glioma, glioblastoma multiforme and craniopharyngioma type of brain tumors.

Convolutional neural networks: CNNs achieve better classification accuracy on large scale datasets due to their capability of joint feature and classifier learning (Shen et al., 2015). The convolutional layer plays a vital role in the operation of CNN. The layers parameters focus around the use of learnable kernels. These kernels are usually small in spatial dimensionality, but spreads along the entirety of the depth of the input. When the data hits a convolutional layer, the layer convolves each filter across the spatial dimensionality of the input to produce a $2 \mathrm{D}$ activation map.

The fully-connected layer contains neurons of which are directly connected to the neurons in the two adjacent layers, without being connected to any layers within them. This is analogous to way that neurons are arranged in traditional forms of ANN. Pooling layers reduce the dimensionality of the representation, and thus further reduce the number of parameters and the computational complexity of the model.

\section{Results and Discussion}

We have collected 26 cases of brain tumor from the Department of Radiology, Rajah Muthiah Medical College Hospital (RMMCH), Chidambaram. All the input data set used for tumor detection consisted of T2-weighted of $512 \times 512$ MR brain images. The MR brain images collected from the patients were acquired on 1.5 Telsa, Intera MR Scanners. This section portrays some experimental results on real data on brain MRI. The number of MR brain images in the input data set is 110 abnormal brain images of astrocytoma, medulloblastoma, glioma, glioblastoma multiforme and craniopharyngioma. The abnormal brain image set consists of images of brain affected by a brain lesion. In the first stage, noise is suppressed using an image filtering. The input MR brain images for astrocytoma, medulloblastoma, craniopharyngioma, glioma and glioblastoma multiforme are shown in Figure 2 (a-e). In the second stage, features are extracted from first order statistics and GLCM. In this research work, nine features are extracted. They are mean, standard deviation, skewness and kurtosis of the first order statistics features. The GLCM based texture features are contrast, correlation, energy, homogeneity and entropy.

Finally, KNN, ANN, SVM and CNN are used to classify the type of tumor images. We evaluate the performance of the classifiers in terms of sensitivity (also called recall in some fields), specificity and accuracy. The formulae for these are given in eq. (6) and eqs. (7)-(8). The three terms are defined as follows: Sensitivity (true positive fraction) is the probability that a diagnostic test is positive and it states that the person has the tumor disease, Specificity (true negative fraction) is the probability that a diagnostic test is negative and that the person does not have the disease. 

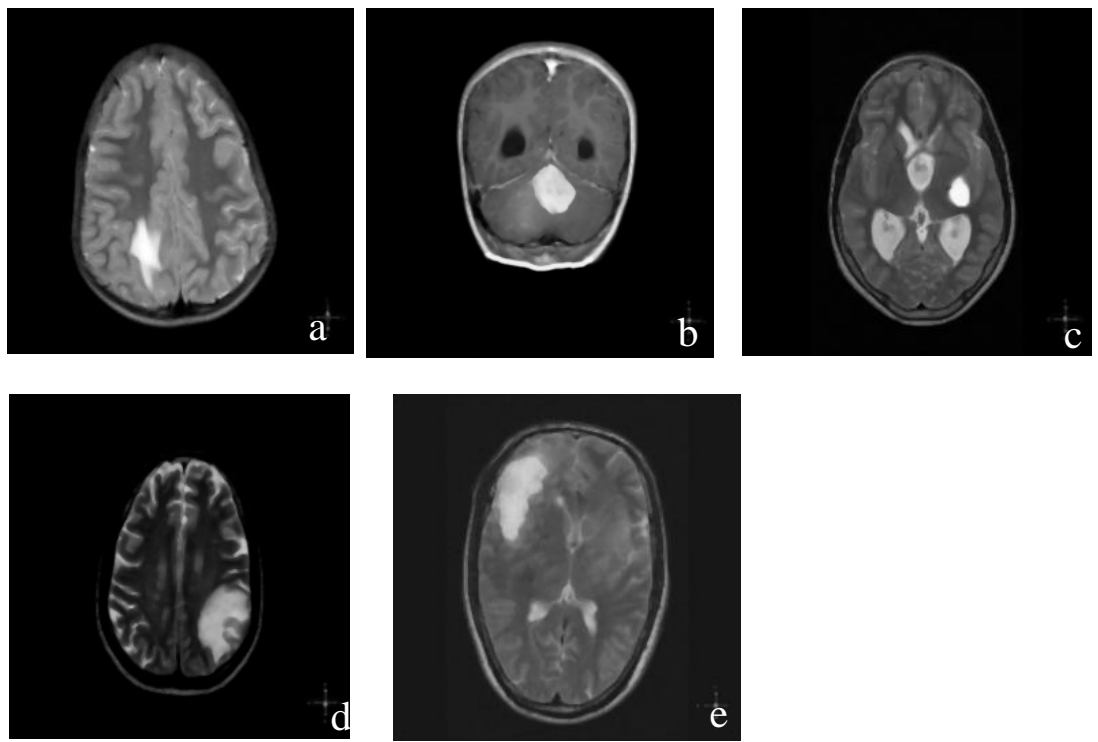

Figure 2. Input MR brain images (a-e)

Table 1

GLCM Features of test samples

\begin{tabular}{cccccc}
\hline \multirow{2}{*}{$\begin{array}{c}\text { Image } \\
\text { No. }\end{array}$} & Contrast & Correlation & Energy & Homogeneity & Entropy \\
\cline { 2 - 5 } & 0.008566 & 0.97740 & 0.6123 & 0.995717 & 2.38500 \\
2 & 0.005587 & 0.968634 & 0.8163 & 0.997207 & 2.98339 \\
3 & 0.005338 & 0.978297 & 0.7487 & 0.997331 & 2.77234 \\
4 & 0.005214 & 0.976312 & 0.7746 & 0.997393 & 2.91287 \\
5 & 0.006642 & 0.973369 & 0.7439 & 0.996679 & 2.68715 \\
6 & 0.005742 & 0.983522 & 0.6463 & 0.997129 & 2.05357 \\
7 & 0.008752 & 0.956693 & 0.7892 & 0.995624 & 2.57759 \\
8 & 0.004593 & 0.969224 & 0.8461 & 0.997703 & 2.77483 \\
9 & 0.009621 & 0.976871 & 0.5744 & 0.99518 & 2.23901 \\
10 & 0.00748 & 0.982728 & 0.5598 & 0.99626 & 2.81441 \\
11 & 0.0072 & 0.980558 & 0.6224 & 0.99640 & 3.11189 \\
12 & 0.00807 & 0.977549 & 0.6325 & 0.99596 & 3.16398 \\
13 & 0.00987 & 0.974196 & 0.6077 & 0.99506 & 2.65219 \\
14 & 0.013532 & 0.969236 & 0.5467 & 0.99323 & 2.42520 \\
15 & 0.015456 & 0.9312 & 0.7601 & 0.99227 & 2.70700 \\
\hline
\end{tabular}

Table 2

First Order Statistics Features of test sample

\begin{tabular}{ccccc}
\hline Image & \multicolumn{4}{c}{ First Order Statistics Feature } \\
\cline { 2 - 5 } No. & Mean & Standard Deviation & Skewness & Kurtosis \\
\hline 1 & 0.006 & 0.01080. & 2.017780 & 6.76021 \\
2 & 0.013 & 0.01896 & 1.384442 & 3.37708 \\
3 & 0.009 & 0.01276 & 1.655632 & 4.73059 \\
4 & 0.011 & 0.01323 & 1.244131 & 3.33805 \\
5 & 0.011 & 0.01910 & 2.274177 & 7.10785 \\
\hline
\end{tabular}

Rajini N, H. (2017). Automatic classification of MR brain tumor images using KNN, ANN, SVM and CNN. International Research Journal of Engineering, IT \& Scientific Research, 3(1), 36-44. Retrieved from https://sloap.org/journals/index.php/irjeis/article/view/895 


\begin{tabular}{ccccc}
\hline 6 & 0.006 & 0.01030 & 1.605176 & 4.28244 \\
7 & 0.008 & 0.01071 & 1.578003 & 4.13867 \\
8 & 0.010 & 0.01203 & 0.826578 & 2.47775 \\
9 & 0.011 & 0.01429 & 0.945757 & 2.65592 \\
10 & 0.010 & 0.00814 & 0.419944 & 2.28365 \\
11 & 0.014 & 0.01945 & 2.495946 & 12.0050 \\
12 & 0.012 & 0.01388 & 1.159288 & 3.16243 \\
13 & 0.014 & 0.02124 & 2.216729 & 9.24762 \\
14 & 0.008 & 0.01097 & 1.308686 & 4.27185 \\
15 & 0.009 & 0.01377 & 2.758114 & 11.6741 \\
\hline
\end{tabular}

Recall or Sensitivity $=\frac{T P}{T P+F N}$

Specificity $=\frac{T N}{T N+F P}$

Accuracy is the probability that a diagnostic test is correctly performed.

$$
\text { Accuracy }=\frac{T P+T N}{T P+T N+F P+F N}
$$

A classification with an accuracy of $89 \%, 90 \%, 91 \%$ and $95 \%$ has been obtained by, k-nearest neighbor, artificial neural network, support vector machine and convolutional neural networks. The proposed method has been evaluated on a data set of 26 patients. The classification performance of the proposed method is tested at slice level. The performance of our algorithm is excellent. The application of the proposed method for tracking tumor is demonstrated to help pathologists distinguish its type of tumor. GLCM and first order statistics feature values are given in Table 1 and 2 . Table 3 presents the performance of classifiers at the slice level.

Table 3

Classification Accuracy for the used classifiers

\begin{tabular}{ccccc}
\hline Classifier & KNN & ANN & SVM & CNN \\
\hline Accuracy & $89 \%$ & $90.11 \%$ & $91.11 \%$ & $95 \%$ \\
Error & $11 \%$ & $7.89 \%$ & $8.89 \%$ & $5 \%$ \\
\hline
\end{tabular}

\section{Conclusion}

Thus, an automated method for classification of five different types of tumors in brain MR images based on first order statistics and gray level co-occurrence matrix is developed. This system has been successfully tested on large brain images causing brain tumor. The proposed system helps the physicians to know about the type of brain tumors, for further treatment. A classification with an accuracy of $89 \%, 90 \%, 91 \%$ and $95 \%$ has been obtained by, k-nearest neighbor, artificial neural network, support vector machine and convolutional neural networks. The system can be designed to classify other types of cancers as well with few modifications. 
Conflict of interest statement and funding sources

The author declared that she has no competing interests.

Statement of authorship

The author have a responsibility for the conception and design of the study. The author have approved the final article.

Acknowledgments

I am grateful to two anonymous reviewers for their valuable comments on the earlier version of this paper.

Rajini N, H. (2017). Automatic classification of MR brain tumor images using KNN, ANN, SVM and CNN. International Research Journal of Engineering, IT \& Scientific Research, 3(1), 36-44. Retrieved from https://sloap.org/journals/index.php/irjeis/article/view/895 


\section{References}

Ahmed, K., Karim, G., Mohamed, B. M., Nacera, B. \& Mohamed, A. (2010). A hybrid approach for automatic classification of brain MRI using genetic algorithm and support vector machine. Leonardo Journal of Sciences, $17,71-82$

Baxt, T. W. G. (1995). Application of Artificial Neural Networks to Clinical Medicine. Lancet, 346, 1135-1138.

Chawla, M., Sharma, S., Sivaswamy, J., Kishore, L. T. (2009). A method for automatic detection and classification of stroke from brain CT images. In: EMBC 2009, Annual International Conference of the IEEE, 3581-3584.

Cover, T.M \& Hart, P.E. (1967). Nearest neighbour pattern classification. IEEE Transactions on Information Theory, $13(1), 21-27$.

Cristianini, N. \& Shawe Taylor, J. (2000). An Introduction to Support Vector Machines and Other Kernel-based Learning Methods. First Edition, Cambridge University Press, England.

Dipali, M. J., Rana, N. K., \& Misra, V. M. (2010). Classification of Brain Cancer Using Artificial Neural Network. In: Proceedings of the IEEE 2nd International Conference on Electronic Computer Technology, 112-116.

Doi, K. (2005). Current status and future potential of computer-aided diagnosis in medical Imaging. British Journal of Radiology, (Spl issue), S3-S19.

Fazel Zarandi, M. H., Zarinbal, M. \& Izadi, M. (2011). Systematic image processing for diagnosing brain tumors: A Type-II fuzzy expert system approach. Applied Soft Computing, 11, 285-294.

Gong, T, Li, S., Wang, J., Lim, C., Pang, T. B. C., Tchoyoson, Lim, C. C., Lee Qi Tian \& C. K., Zhang, Z. (2011). Automatic labeling and classification of brain CT images. In: 18th IEEE International Conference on image Process, 1581-1584.

Haralick, R. M., Shanmugam, K \& Dinstein, I. (1973). Textural features for image classification, IEEE Transactions on Systems, Man, and Cybernetics, SMC, 36 (1973) 610-621.

Hema Rajini, N \& Bhavani, R. (2014). Automatic classification of computed tomography brain images using ANN, k-NN and SVM. AI and Society, 29(1), 97-102.

Padma, A. \& Sukanesh, R. (2011). Automatic classification and segmentation of brain tumor in CT images using optimal dominant gray level run length texture features. International Journal of Advanced Computer Science and Applications, 2(10), 53-59.

Shen, W., Zhou, M., Yang, F., Yang, C. \& Tian, J. (2015). Multi-scale convolutional neural networks for lung nodule classification, In: Proceedings of 24th International Conference on Information Processing in Medical Imaging, 588-599.

Zhang, W. L. \& Wang, X. Z. (2007). Feature extraction and classification for human brain CT images. In: Proceedings of the IEEE international conference on machine learning and cybernetics, 2, 19-22. 